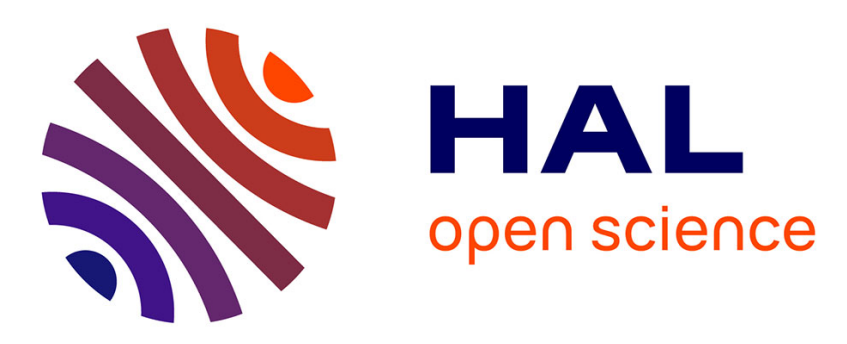

\title{
Preparation of Swellable Hydrogel-Containing Colloidosomes from Aqueous Two-Phase Pickering Emulsion Droplets
}

\author{
Jean-Paul Douliez, Nicolas Martin, Thomas Beneyton, Jean-Charles Eloi, \\ Jean-Paul Chapel, Laurence Navailles, Jean-Christophe Baret, Stephen Mann, \\ Laure Beven
}

\section{To cite this version:}

Jean-Paul Douliez, Nicolas Martin, Thomas Beneyton, Jean-Charles Eloi, Jean-Paul Chapel, et al.. Preparation of Swellable Hydrogel-Containing Colloidosomes from Aqueous Two-Phase Pickering Emulsion Droplets. Angewandte Chemie International Edition, 2018, 57 (26), pp.7780-7784. 10.1002/anie.201802929 . hal-02119101

\section{HAL Id: hal-02119101 \\ https://hal.science/hal-02119101}

Submitted on 30 Mar 2021

HAL is a multi-disciplinary open access archive for the deposit and dissemination of scientific research documents, whether they are published or not. The documents may come from teaching and research institutions in France or abroad, or from public or private research centers.
L'archive ouverte pluridisciplinaire HAL, est destinée au dépôt et à la diffusion de documents scientifiques de niveau recherche, publiés ou non, émanant des établissements d'enseignement et de recherche français ou étrangers, des laboratoires publics ou privés. 
Douliez, J. P., Martin, N., Beneyton, T., Eloi, J. C., Chapel, J. P., Navailles, L., Baret, J. C., Mann, S., \& Béven, L. (2018). Preparation of Swellable Hydrogel-Containing Colloidosomes from Aqueous TwoPhase Pickering Emulsion Droplets. Angewandte Chemie International Edition, 57(26), 7780-7784. https://doi.org/10.1002/anie.201802929

Peer reviewed version

Link to published version (if available):

10.1002/anie.201802929

Link to publication record in Explore Bristol Research

PDF-document

This is the author accepted manuscript (AAM). The final published version (version of record) is available online via Wiley at https://onlinelibrary.wiley.com/doi/abs/10.1002/anie.201802929. Please refer to any applicable terms of use of the publisher.

\section{University of Bristol - Explore Bristol Research General rights}

This document is made available in accordance with publisher policies. Please cite only the published version using the reference above. Full terms of use are available: http://www.bristol.ac.uk/red/research-policy/pure/user-guides/ebr-terms/ 


\title{
Preparation of swellable hydrogel-containing colloidosomes from aqueous two-phase Pickering emulsion droplets
}

\author{
Jean-Paul Douliez, ${ }^{[a]}$ Nicolas Martin, ${ }^{[b]}$ Thomas Beneyton, ${ }^{[c]}$ Jean-Charles Eloi, ${ }^{[d]}$ Jean-Paul Chapel, ${ }^{[c]}$ \\ Laurence Navailles, ${ }^{[c]}$ Jean-Christophe Baret, ${ }^{[c]}$ Stephen Mann, ${ }^{[b]}$ and Laure Béven, ${ }^{[a]}$
}

Dedication ((optional))

\begin{abstract}
We describe the fabrication of stable colloidosomes derived from water-in-water Pickering-like emulsions produced by addition of fluorescent amine-modified polystyrene latex beads to an aqueous two-phase system consisting of dextran-enriched droplets dispersed in a PEG-enriched continuous phase. Addition of polyacrylic acid followed by carbodiimide-induced crosslinking with dextran produces hydrogelled droplets capable of reversible swelling and selective molecular uptake and exclusion. Colloidosomes produced specifically in all-water systems could offer new opportunities in microencapsulation and the bottom-up construction of synthetic protocells.
\end{abstract}

Colloidosomes are microcapsules delineated by a semipermeable shell formed by crosslinked colloidal particles. ${ }^{[1]}$ Their production generally starts from colloidal particle-stabilized water-inoil (w/o) or oil-in-water (o/w) Pickering emulsions, ${ }^{[2]}$ which are metastable upon centrifugation, removal of the o/w phase or transfer into different solvents. Crosslinking of the interfacial colloidal particles provides a solid shell at the w/o interface that converts the Pickering emulsion into robust capsules called colloidosomes ${ }^{[3]}$. An alternative approach involves using an aqueous gel as the internal phase ${ }^{[4,5]}$. Hydrogels are of considerable interest for capturing biomolecules and cells. ${ }^{[6]}$ Colloidosomes can encapsulate and subsequently release active ingredients, which makes them not only of high interest for microencapsulation purposes but also as minimal forms of artificial cells. ${ }^{[1,7]}$ Control of the permeability of the shell, which originates from the interstices existing between the colloidal particles, is of crucial importance for these applications, especially when small bioactive molecules are concerned. [4, 8] Robust approaches to retain such payloads within colloidosomes, such as interactions within their lumen or impermeabilisation of the shell, still need to be developed. ${ }^{[1]}$

To date, encapsulation of water-soluble biomacromolecules in the lumen of colloidosomes has essentially relied on the addition of the solutes in the water phase of a w/o Pickering emulsion before crosslinking the interfacial particles. This implies that all ingredients have to be initially encapsulated since the composition cannot be further modified. During the cross-linking process, the cross-linkers may diffuse and react with the encapsulated biomolecules.

[a] Dr J-P Douliez and Dr L. Béven UMR 1332, biologie du fruit et pathologie, INRA, Univ. Bordeaux, centre de Bordeaux, 33883 Villenave d'Ornon, France

E-mail: jean-paul.douliez@inra.fr

[b] Dr N. Martin and Prof. S. Mann Centre for Organized Matter Chemistry and Centre for Protolife Research, School of Chemistry, University of Bristol, Cantock's Close, BS8 1TS Bristol, UK
Moreover, transfer of colloidosomes into water usually involves the use of organic solvents to remove the continuous oil phase. These features can alter the properties of the encapsulated biomolecules. Preparing colloidosomes via water-in-water (w/w) emulsions that are capable of spontaneous biomolecular uptake would then be of immediate practical value. This has been suggested a decade ago, using fat or protein particles in dextran/methyl cellulose aqueous two-phase systems but the uptake of payloads was not studied. ${ }^{[9]}$ In principle, w/w emulsions produced by liquid-liquid phase separation ${ }^{[10]}$ can be used to uptake payloads because the resulting droplets can spontaneously sequester biomolecules. ${ }^{[11,12]}$ Liquid-liquid phase separation has been achieved in a variety of systems, including incompatible polymers (aqueous two-phase systems, ATPS), [11, 13] counter-charged molecules (complex coacervation) ${ }^{[14]}$ or surfactants [15] (surfactant complexation), and offers exciting possibilities to build protocells and bioreactors. As with w/o and o/w, w/w emulsions need to be stabilized, otherwise phase separation occurs after a short period of time (minutes to hours). This task has recently attracted growing interest and there are now a large number of ingredients available to form Pickering-like emulsion droplets. ${ }^{[2,9,16,17]}$ However, these are not stable, being disassembled upon dilution below the concentration of ATPS formation.

In this work, we report the production of stable colloidosomes derived from w/w Pickering-like emulsions produced by addition of fluorescent amine-modified polystyrene latex beads (LB) to an ATPS consisting of dextran-enriched droplets dispersed in a PEG-enriched continuous phase. The droplet core is then gelled to form molecularly crowded colloidosomes capable of reversibly swelling and spontaneous uptake/exclusion of payloads, depending on the electrostatic interactions and the swelling properties of the colloidosomes (see figure SC1). Our results suggest that the formation of colloidosomes specifically in all-water systems could offer new opportunities in microencapsulation and bottom-up synthetic biology.

Dextran (2,000 kDa, $4 \mathrm{mg} / \mathrm{mL})$-enriched droplets suspended in a PEG-enriched (20 kDa, $70 \mathrm{mg} / \mathrm{mL}$ ) continuous phase were stabilized against coalescence by addition of $100 \mathrm{~nm}$-sized fluorescent aminemodified latex beads to produce a Pickering-like $\mathrm{w} / \mathrm{w}$ emulsion (figure S1). Both PEG and dextran are expected to adsorb on the LBs, which decrease even further the colloidosomes/solution free energy through the contact angle of the particles with the interface as already described in the literature. ${ }^{[1,2]}$ The droplets had an average size of ca. $15 \mu \mathrm{m}$, with a polydispersity of $+/-10 \mu \mathrm{m}$. As expected, dilution of this emulsion with water below the level required for formation of the ATPS resulted in disassembly of the emulsion droplets (figure S1).

[c] Dr T. Beneyton, Dr L. Navailles, Prof. J-P Chapel and Prof. J-C Baret CNRS, Univ. Bordeaux, CRPP, 115 Av. A. Schweitzer, 33600 Pessac, France

[d] Dr JC Eloi Chemical Imaging Facility, School of Chemistry, University of Bristol, Cantock's Close, BS8 1TS Bristol, UK 

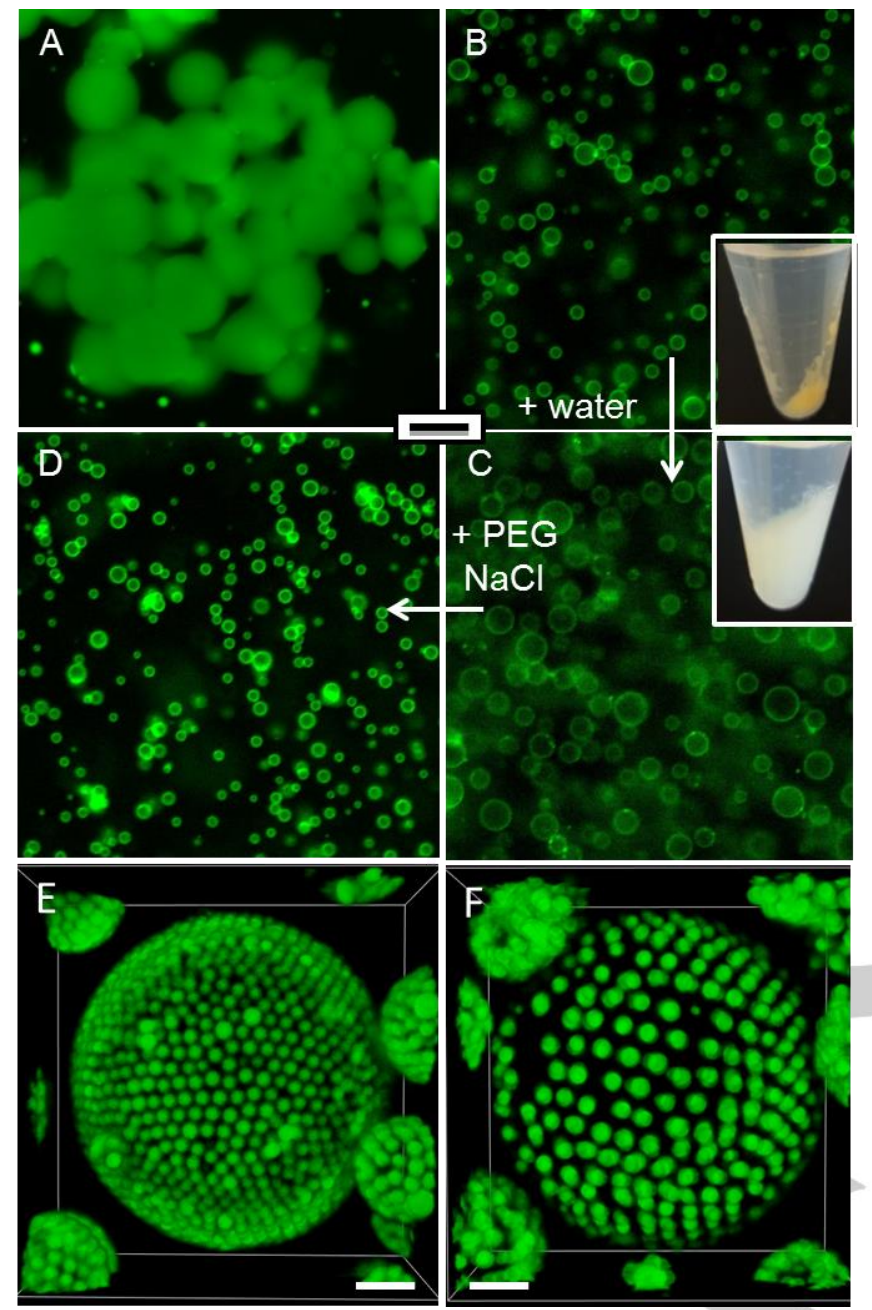

Figure 1. Epifluorescence images (scale bar $=50 \mu \mathrm{m}$ ) of A) Aggregated hydrogelled droplets produced by mixing dextran-in-PEG droplets in the presence of PAA and EDC. B) Hybrid PAA/dextran hydrogel-colloidosomes produced by using the same mixture as above but pre-stabilized using latex beads. The inset shows a photo of the sample tube after centrifugation. $\mathrm{C}$ ) Same as B) but observed after dilution in water (2 times), illustrating swelling of the hydrogelled colloidosomes. The inset shows a photo of the sample tube after centrifugation. D) Same as C) but observed after re-addition of PEG (7\%) and $\mathrm{NaCl}(20 \mathrm{mM})$, illustrating reversible shrinking of the swollen hydrogelled colloidosomes. E,F) Confocal images of two different hybrid hydrogelcolloidosomes produced by using $1 \mu \mathrm{m}$ latex beads; E) in the pristine (relaxed) form and F) upon swelling (scale bar $=5 \mu \mathrm{m}$ ).

To prevent disassemby of the Pickering emulsion, our first intention was to cross-link the amine-modified latex beads using polyacrylic acid (PAA) and water-soluble carbodiimide (EDC: 1-ethyl3-(3-dimethylaminopropyl)carbodiimide) to form colloidosomes. However, in a control experiment, we observed that addition of PAA $\left(5,100 \mathrm{~g} / \mathrm{mol}, \mathrm{Na}^{+}\right.$salt $)$and EDC to a latex bead-free dextran-in-PEG emulsion resulted in the formation of aggregated hydrogelled droplets (figure 1A). We attributed this to the covalent coupling of the carboxylate groups of PAA chains to the alcohol-bearing saccharide

units of the dextran molecules (Figure S2). Both these polymers are concentrated within the droplets making the esterification reaction possible, although dehydration reactions are not favored in water. ${ }^{[18}$ Here, aggregation occurred due to partial coalescence during the esterification reaction. Addition of latex beads prevented droplet aggregation and afford colloidosomes with a hydrogelled core with a diameter of ca. $12 \mu \mathrm{m}(+/-7 \mu \mathrm{m})$. (figure $1 \mathrm{~B})$. In contrast to the Pickering emulsion, the capsules remained intact upon dilution with water but exhibited a 2-fold increase in their size $(d=22+/-5 \mu \mathrm{m}$, figure $1 \mathrm{C}$ ). This volume expansion was attributed to swelling of the internal dextran/PAA hydrogel induced by the decrease of the osmotic pressure and electrostatic repulsions between PAA chains. The colloidosomes recovered their initial size upon re-dispersion with both PEG (7\%) and $\mathrm{NaCl}(20 \mathrm{mM}$, figure 1D), whereas PEG $(7 \%)$ or $\mathrm{NaCl}(20 \mathrm{mM})$ alone produced swollen colloidosomes (figure S3). These results demonstrate that the swelling phenomenon is reversible by tuning the composition of the outer phase.
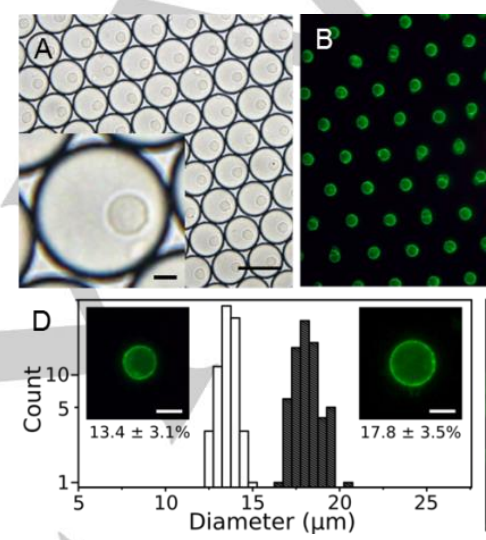

Figure 2. Microfluidic fabrication of monodisperse hybrid PAA/dextran hydrogel colloidosomes. A) White light and B) epifluorescence images of 40 pL w/o droplets containing single hydrogelled colloidosomes. C) Epifluorescence image after picoinjection of water (3 fold dilution). D) Size distribution of both pristine (white) and swollen (black) colloidosomes. E) Epifluorescence image of colloidosomes extracted from the w/o emulsion, centrifuged and resuspended in PEG (7\%). Scale bars are $50 \mu \mathrm{m}(10 \mu \mathrm{m}$ in all insets).

Hydrogelled colloidosomes were also successfully produced with latex beads having a lower or a higher diameter $(50 \mathrm{~nm}, 1$ or $2 \mu \mathrm{m}$, figures $1 E, F$ and S4). Interfacial localization of the largest latex beads, as well as the swelling effect upon dilution in water were clearly observed since the latex beads became separated from each other upon dilution. This also showed that the beads were not crosslinked together, but chemically linked to the underlying dextran/PAA hydrogel (figure S4).

In the context of bottom-up synthetic biology, the construction of populations of identical and isolated synthetic protocells is of strong interest. Droplet-based microfluidics has been widely used to increase the monodispersity of compartments prepared from hydrogels and ATPS. [17, 19] We used this technology to produce hybrid hydrogel-containing colloidosomes in a highly controlled manner within w/o droplets. The w/w droplets (Dex/PEG) covered by LBs were produced in $40 \mathrm{pL}$ w/o droplets and synthesis of PAA/dextran hydrogelled colloidosomes occurred in situ upon 
addition of both PAA and EdC (figure S5). Single colloidosomes were obtained in every $w / 0$ droplet, with a high monodispersity $(\mathrm{d}=$ $13.4+/-0.4 \mu \mathrm{m}$ ) (figures $2 \mathrm{~A}, \mathrm{~B}$ and D). W/o droplets were then picoinjected with water to study the stability and swelling properties of the hydrogelled colloidosomes. As shown in bulk experiments, colloidosomes prepared without gellification were destructed upon dilution (Figure S6). Oppositely, hydrogelled colloidosomes resisted to dilution and exhibited swelling with a $\sim 1.4$-fold increase in size ( $\mathrm{d}$ $=17.8+/-0.6 \mu \mathrm{m}$ ) (figures $2 \mathrm{C}$ and $\mathrm{D}$ ). Interestingly, after triggered coalescence of the w/o emulsion matrix, hydrogelled colloidosomes were centrifuged and re-suspended in aqueous media (figure $2 \mathrm{E}$ ). These results confirm the efficient crosslinking of the hydrogelled colloidosomes and their swelling properties. Then, microfluidics allows the formation of highly monodisperse structures that can be further manipulated in situ or in water.
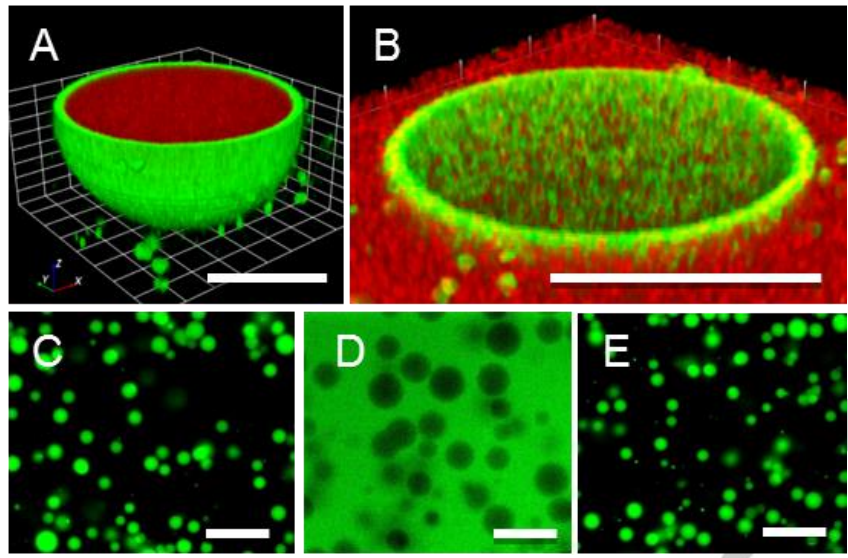

Figure 3. Uptake and exclusion of macromolecular payloads by PAA/dextran hydrogelled colloidosomes. Confocal images of A) pristine (relaxed) hydrogel-containing colloidosomes showing uptake of RITC-dextran $(70 \mathrm{kDa})$ (scale bar, $5 \mu \mathrm{m}$ ) and B) swollen colloidosomes showing exclusion of RITC-dextran (70kDa) (scale bar, $20 \mu \mathrm{m}$ ). Epifluorescence images (scale bar, $50 \mu \mathrm{m}$ ) of C) pristine colloidosomes in the relaxed state showing uptake of FITC-dextran $(500 \mathrm{kDa}), \mathrm{D}$ ) expanded (3 fold dilution in water) colloidosomes showing exclusion of FITC-dextran $(500 \mathrm{kDa})$ and E) Shrinking of expanded colloidosomes upon addition of PEG $(7 \%)$ and $\mathrm{NaCl}(20 \mathrm{mM})$ showing re-entry of FITC-dextran $(500 \mathrm{kDa})$.

Interestingly, sequestration of payloads strongly correlated with the swelling state of the hydrogelled colloidosomes. Notably, RITCdextran $(70 \mathrm{kDa})$ was spontaneously sequestered in the pristine gelled (relaxed) colloidosomes but fully excluded from the swollen capsules (figures $3 \mathrm{~A}$ and $\mathrm{B}$ ). This feature was also clearly evidenced when using a high molecular weight FITC-dextran (500 kDa), which also indicated that shrinking of the expanded colloidosomes restored the uptake properties Figures $3 \mathrm{C}, \mathrm{D}$ and $\mathrm{E})$. The results suggest that large polymers can diffuse through the interstices of the closely packed latex bead shell (interstitial size, ca. $0.7 d$, where $d=$ bead diameter $\left.{ }^{[1]}\right)$, even in the relaxed colloidosomes. Once across the shell, the macromolecules readily diffuse throughout the $\mathrm{PAA} /$ dextran hydrogel matrix encapsulated within the colloidosomes. In contrast, expansion of the hydrogel results in exclusion of fluorescent dextrans, which bear negative charges on the fluorescent moiety. We attribute this to the electrostatic repulsions between these charges and the PAA/dextran hydrogel, since the ionic strength decreased upon dilution. Whereas partitioning of payloads have been shown to depend on the $\mathrm{pH}$ in ATPS, ${ }^{[20]}$ we demonstrated that it may be tuned in our colloidosomes by the composition of the outer phase.

We questioned if electrostatic interactions between swollen colloidosomes and payloads would also afford sequestration. Replacing the slightly negatively charged FITC-dextran with FITCCM-dextran resulted in complete exclusion from the expanded colloidosomes (Figure 4A). In contrast, positively charged FITCDEAE-Dextran $(150 \mathrm{kDa})$ was sequestered confirming that electrostatic forces drive the interaction (Figure 4B). We were able to reverse these differences in uptake/exclusion properties by adding a hyper-branched positively charged polyethylene imine (HB-PEI, 25 $\mathrm{kDa}$ ) to expanded hydrogelled colloidosomes to produce colloidosomes with a positively charged gelled core (after extensive washing). The resulting colloidosomes then uptake FITC-CMdextran $(150 \mathrm{kDa})$ but exclude positively charged DEAE-FITCdextran (150 kDa) (figures $4 \mathrm{C}$ and D). Finally, we took advantage that HB-PEI has been used as nanocontainers to phase transfer small dyes ${ }^{[21]}$ to show that fluorescein and Congo red were also sequestered within the HB-PEI-modified colloidosomes (figure S7).

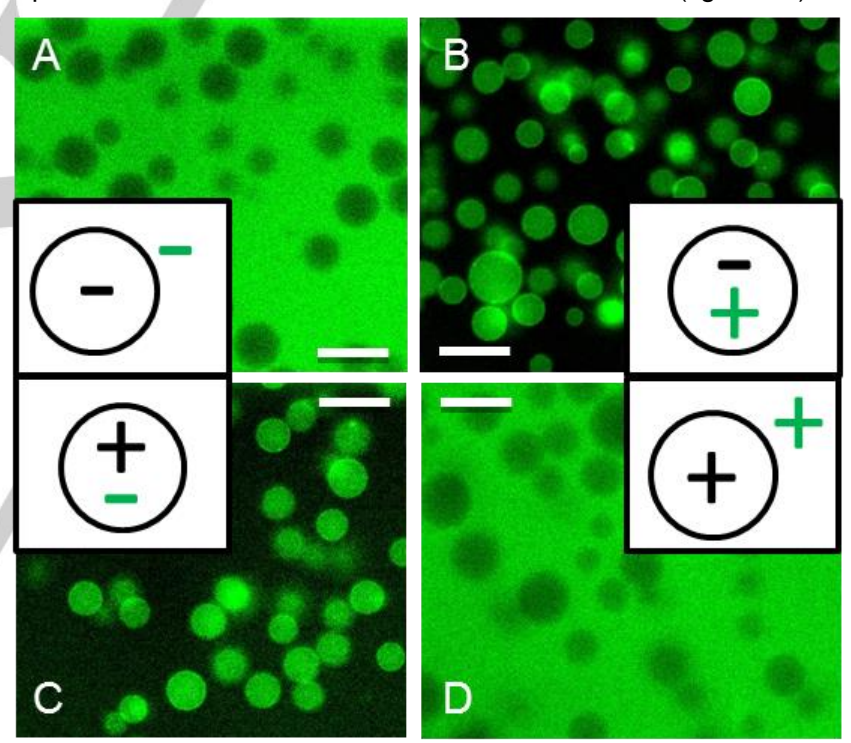

Figure 4. Uptake and exclusion of payloads by swollen PAA/dextran hydrogel-containing colloidosomes. Epifluorescence images of swollen colloidosomes in the presence of A) CM-Dex-FITC (150kDa) and B) DEAEDex-FITC (150kDa) showing exclusion and uptake, respectively. $C$ and $D$ ) swollen hydrogel-colloidosomes pre-treated with HB-PEI showing uptake of CM-Dex-FITC (150kDa) and exclusion of DEAD-Dex-FITC (150kDa), respectively. The insets represent schematic images of a colloidosome with negatively $(A$ and $B)$ or positively $(C$ and $D)$ charged cores in the presence of positively ( $A$ and $C$ ) or negatively charged dextran (green) ( $B$ and $D)$. (scale bar stands for $50 \mu \mathrm{m}$ for all images)

In summary, we report the one-pot fabrication in water of a new generation of colloidosomes by using an ATPS, circumventing the use of oil and organic solvents. The droplets are stabilized by the spontaneous interfacial adsorption of amine-modified latex beads (w/w Pickering emulsions) and core gelation to produce colloidosomes with molecular crowded interiors that can reversibly 
swell. These colloidosomes are easily prepared in bulk but also via microfluidics, affording monodisperse objects and eventually, manipulation of single colloidosomes. We develop a novel hydrogel from an in situ esterification reaction in water within the colloidosome core, which occurs because of the molecular crowding associated with the ATPS. The colloidosomes are produced with latex beads of variable diameter and it should be possible to extended our approach to other ATPS stabilized by vesicles, proteins or polymers. ${ }^{[2,16,17]}$ Moreover, cross-linking beads using glutaraldehyde or derivatives ${ }^{[1]}$ should also allow the production of colloidosomes in such ATPS. An additional advantage of the colloidosomes described herein compared to those prepared from conventional w/o emulsions, is that they can spontaneously uptake certain payloads after their formation. This attractive feature is based on the intrinsic properties of ATPS, which have long been used to sequester a range of biomolecules. The full exclusion and re-entry of the payloads within colloidosomes is triggered by varying the composition of the continuous phase. Payloads are also sequestered under non-ATPS conditions by driving electrostatic interactions within the colloidosome core, suggesting plausible applications for microencapsulation. In the longer term, the use of such colloidosomes for controlled payload release requires further advances in design. For instance, pores between the latex beads could be blocked by using gates ${ }^{[7]}$ or the layer-by-layer technique to trigger payload release ${ }^{[22]}$. Alternatively, we also show that the use of nanocontainers ${ }^{[21]}$ affords efficient phase transfer of small dyes from water to colloidosomes. Finally, colloidosomes prepared from w/o emulsions have been recently used as protocell models, suggesting that the micro-compartments prepared by the droplet stabilization of ATPS colloidosomes could be useful constructs in areas of bottom-up synthetic biology.

\section{Experimental Section}

Experimental Details and additional scheme and figures can be found in Supporting information

Acknowledgements: JPD would like to thank N. Coudon and $M$. Li for preliminary experiments. TB and JCB acknowledge the financial support by the ERC (FP7/2007-2013 /ERC Grant agreement 306385Sofl) and by the Network Max Syn Bio. The epifluorescence microscopy imaging was performed at the Bordeaux Imaging Center, member of the FranceBiolmaging national infrastructure (ANR-10-INBS-04). NM and SM acknowledge the ERC Advanced Grant Scheme (ADG 740235) and Wolfson Bioimaging Facility for access to confocal fluorescence microscopy.

Keywords: Colloidosomes • Aqueous two-phase systems • Sequestration $\bullet$ encapsulation $\cdot$ water-in-water emulsion
[1] K. L. Thompson, M. Williams, S. P. Armes, J. Coll. Inter. Sci. 2015, 447, 217-228.

[2] B. P. Binks, Langmuir 2017, 33, 6947-6963

[3] O. D. Velev, K. Nagayama, Langmuir 1997, 13, 1856-1859; A. D. Dinsmore, M. F. Hsu, M. G. Nikolaides, M. Marquez, A. R. Bausch, D. A. Weitz, Science 2002, 298, 1006-1009.

[4] O. J. Cayre, J. Hitchcock, M. S. Manga, S. Fincham, A. Simoes, R. A. Williams, S. Biggs, Soft Matt. 2012, 8, 4717-4724.

[5] P. F. Noble, O. J. Cayre, R. G. Alargova, O. D. Velev, V. N. Paunov, J. Amer. Chem. Soc. 2004, 126, 8092-8093.

[6] X. T. Le, L.-E. Rioux, S. L. Turgeon, Adv. Coll. Inter. Sci. 2017, 239, 127135; A. Matalanis, D. J. McClements, Food Hydro. 2013, 31, 15-25; D. Suzuki, K. Horigome, T. Kureha, S. Matsui, T. Watanabe, Polym. J. 2017, 49,695 .

[7] M. Li, R. L. Harbron, J. V. M. Weaver, B. P. Binks, S. Mann, Nat. Chem. 2013, 5, 529-536.

[8] H. N. Yow, A. F. Routh, Langmuir 2009, 25, 159-166.

[9] A. T. Poortinga, Langmuir 2008, 24, 1644-1647.

[10] J. Esquena, Cur. Op. Coll. Inter. Sci. 2016, 25, 109119.

[11] W. M. Aumiller Jr, C. D. Keating, Adv. Coll. Inter. Sci. 2017, 239, 75-87.

[12] S. Lindhoud, M. M. A. E. Claessens, Soft Matt. 2016, 12, 408-413; A. I. Oparin, New York, 1953.

[13] C. D. Keating, Acc. Chem. Res. 2012, 45, 2114-2124; C. A. Strulson, R. C. Molden, C. D. Keating, P. C. Bevilacqua, Nat. Chem. 2012, 4, 941-946.

[14] A. J. Dzieciol, S. Mann, Chem. Soc. Rev. 2012, 41, 79-85; S. Koga, D. S. Williams, A. W. Perriman, S. Mann, Nat. Chem. 2011, 3, 720-724.

[15] D. Garenne, L. Beven, L. Navailles, F. Nallet, E. J. Dufourc, J.-P. Douliez, Angew. Chem. Inter. Ed. 2016, 55, 13475-13479; D. Garenne, L. Navailles, F. Nallet, A. Grélard, E. J. Dufourc, J.-P. Douliez, J. Coll. Inter. Sci. 2016 468, 95-102; J.-P. Douliez, N. Martin, C. Gaillard, T. Beneyton, J.-C. Baret, S. Mann, L. Beven, Angew. Chem. Inter. Ed. 2017, 56, 13689-13693; B. W. Barry, G. M. T. Gray, J. Coll. Inter. Sci. 1975, 52, 327-339; M. Wang, Y.

Wang, Soft Matt. 2014, 10, 7909-7919; W. Zhao, Y. Wang, Adv. Coll. Inter. Sci. 2017, 239, 199-212.

[16] D. C. Dewey, C. A. Strulson, D. N. Cacace, P. C. Bevilacqua, C. D. Keating, Nat. Comm. 2014, 5; R. A. de Freitas, T. Nicolai, C. Chassenieux, L. Benyahia, Langmuir 2016, 32, 1227-1232; A. Gonzalez-Jordan, L. Benyahia, T. Nicolai, Coll. Surf. A 2017, 532, 332-341; A. Gonzalez-Jordan, T. Nicolai, L. Benyahia, Langmuir 2016, 32, 7189-7197; B. T. Nguyen, T. Nicolai, L. Benyahia, Langmuir 2013, 29, 10658-10664; B. T. Nguyen, W. Wang, B. R. Saunders, L. Benyahia, T. Nicolai, Langmuir 2015, 31, 36053611; T. Nicolai, B. Murray, Food Hydro. 2017, 68, 157-163; K. R. Peddireddy, T. Nicolai, L. Benyahia, I. Capron, ACS Macro Lett. 2016, 5, 283-286.

[17] A. F. Mason, B. C. Buddingh, D. S. Williams, J. C. M. van Hest, J. Amer. Chem. Soc. 2017, 139, 17309-17312.

[18] K. Manabe, X.-M. Sun, S. Kobayashi, J. Amer. Chem. Soc. 2001, 123 , 10101-10102

[19] B. G. Chung, K.-H. Lee, A. Khademhosseini, S.-H. Lee, Lab on a Chip 2012, 12, 45-59; Z. Zhu, C. J. Yang, Acc. Chem. Res. 2017, 50, 22-31; N.-N. Deng, M. Yelleswarapu, L. Zheng, W. T. S. Huck, J. Amer. Chem. Soc. 2016, 139, 587-590; A. B. Theberge, F. Courtois, Y. Schaerli, M. Fischlechner, C. Abell, F. Hollfelder, W. T. S. Huck, Angew. Chem. Inter. Ed. 2010, 49, 5846-5868; R. Seemann, M. Brinkmann, Th. Pfohl, S Herminghaus, Rep. Prog. Phys. 2012, 75, 016601. L. Zhang, L.-H. Cai, P Lienemann, S., T. Rossow, I. Polenz, Q. Vallmajo-Martin, M. Ehrbar, H. Na D. Mooney, J., D. Weitz, A., Angew. Chem. Int. Ed. 2016, 55, 13470-13474.

[20] L. M. Dominak, E. L. Gundermann, C. D. Keating, Langmuir 2010, 26, 5697-5705.

[21] S.-E. Stiriba, H. Kautz, H. Frey, J. Amer. Chem. Soc. 2002, 124, 96989699; H. Frey, R. Haag, Rev. Mol. Biotech. 2002, 90, 257-267.

[22] D. E. Discher, A. Eisenberg, Science 2002, 297, 967-973.

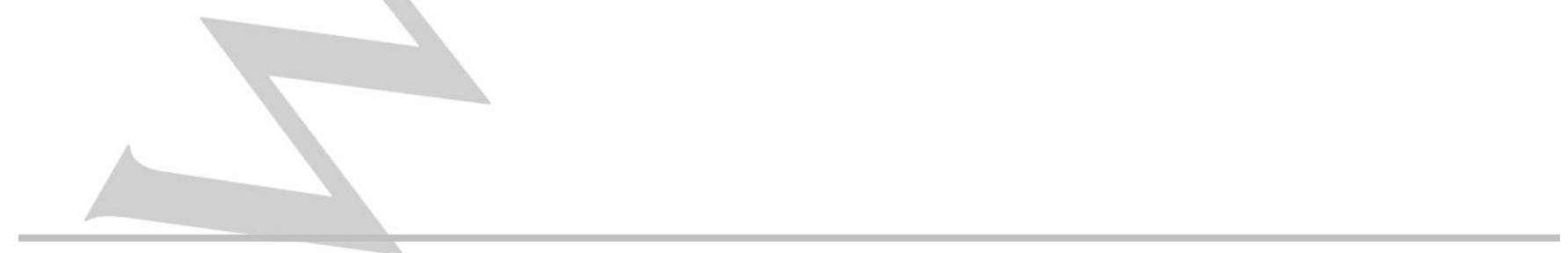


Entry for the Table of Contents (Please choose one layout)

Layout 1:

COMMUNICATION

Hybrid hydrogel-colloidosomes capable of swelling and spontaneous uptake of payloads have been fabricated from water-in-water emulsions.
Jean-Paul Douliez, ${ }^{*[a]}$ Nicolas Martin, ${ }^{[b]}$ Thomas Beneyton, ${ }^{[c]}$ JeanPaul Chapel, ${ }^{[c]}$ Laurence Navailles, ${ }^{[c]}$ Jean-Christophe Baret, ${ }^{[c]}$ Stephen Mann, ${ }^{[b]}$ and Laure Beven ${ }^{[a]}$

Page No. - Page No.

Hybrid colloidosomes-ATPS

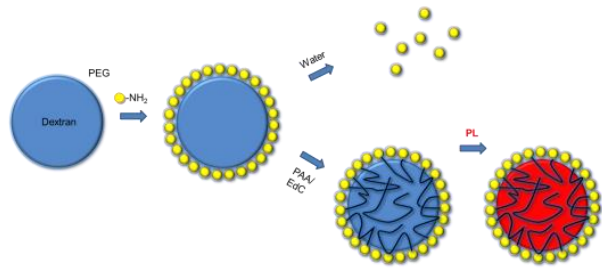

